\section{$\underset{\substack{\text { hommes } \\ \text { \& migrations }}}{ }$}

\section{Hommes \& migrations}

Revue française de référence sur les dynamiques

migratoires

$1316 \mid 2017$

L'islam en Europe

\title{
Soldats et travailleurs portugais en France (1916-1918)
}

\author{
Marie-Christine Volovitch-Tavares
}

\section{(2) OpenEdition}

\section{Journals}

Édition électronique

URL : http://journals.openedition.org/hommesmigrations/3825

DOI : 10.4000/hommesmigrations.3825

ISSN : 2262-3353

\section{Éditeur}

Musée national de l'histoire de l'immigration

\section{Édition imprimée}

Date de publication : 1 mars 2017

Pagination : 164-169

ISBN : 978-2-919040-37-7

ISSN : $1142-852 X$

\section{Référence électronique}

Marie-Christine Volovitch-Tavares, « Soldats et travailleurs portugais en France (1916-1918) », Hommes \& migrations [En ligne], 1316 | 2017, mis en ligne le 01 mars 2017, consulté le 17 septembre 2020. URL : http://journals.openedition.org/hommesmigrations/3825 ; DOI : https://doi.org/10.4000/ hommesmigrations.3825 


\section{SOLDATS ET TRAVAILLEURS PORTUGAIS EN FRANCE (1916-1918)}

Par MARIE-CHRISTINE VOLOVITCH-TAVARES, professeure d'histoire, vice-présidente du Centre d'études et de recherches sur les migrations ibériques (CERMI).

Jusing usqu'à la Première Guerre mondiale, on ne comptait qu'un millier de Portugais en France, des rentiers aisés, des artistes puis, à la fin du $x \mid x^{e}$ siècle, de petits groupes d'exilés républicains réfugiés et quelques dizaines d'ouvriers venus après avoir travaillé avec des entreprises françaises au Portugal.

La Première Guerre mondiale a marqué un tournant radical en amorçant l'immigration portugaise en France. Jusque-là, les Portugais émigraient massivement vers le Brésil et, beaucoup moins, vers d'autres pays d'Amérique ou vers les colonies portugaises, mais ils allaient très rarement en Europe, à l'exception de l'Espagne voisine. C'est l'entrée en guerre du Portugal, le 9 mars 1916, aux côtés de la France et de la Grande-Bretagne et la Convention de maind'œuvre signée le 28 octobre 1916 entre la France et la Portugal qui firent de la France un horizon pour les émigrants portugais. Ainsi, partirent vers la France à la fois les soldats du corps expéditionnaire portugais (CEP) et les travailleurs recrutés dans le cadre de la Convention de maind'œuvre.

Les soldats du corps expéditionnaire portugais (CEP), 56500 hommes, prirent position durant les premiers mois de 1917 sur le front des Flandres (Pas-de-Calais), sous commandement britannique. En même temps, dans le cadre de l'effort de guerre, la Convention de main- d'œuvre permettait à la France de recruter des travailleurs portugais. Le gouvernement français espérait faire venir de 15000 à 20000 ouvriers pour ses usines, ses forêts et ses campagnes.

À la fin de la guerre, une partie des travailleurs portugais restèrent en France. Parallèlement, un certain nombre de soldats ne rentrèrent pas au Portugal et leur exemple poussa des compatriotes à venir y chercher du travail. Cette nouvelle dynamique migratoire ne s'est jamais arrêtée jusqu'à nos jours, même si elle a connu de grandes variations du fait des évolutions économiques et politiques de la France, du Portugal et des conjonctures internationales.

\section{Des travailleurs portugais pour les usines et les campagnes en France}

La guerre exigeait une forte activité économique alors même que la France, faisant déjà appel avant 1914 à de nombreux travailleurs étrangers (Belges, Italiens, Espagnols), devait remplacer les hommes mobilisés et compenser les productions des régions du Nord et de l'Est de la France occupées par l'armée allemande. De- 
puis la fin de l'année 1914, on avait embauché des femmes et des travailleurs venus de pays étrangers (Belges, Espagnols) ou de colonies (Indochinois, Maghrébins), et des Chinois. Dès 1915, des responsables français avaient contacté les autorités portugaises pour organiser le recrutement d'ouvriers dans le cadre légal d'un accord de main-d'œuvre. Le gouvernement portugais avait été réticent, suivant en cela la tradition politique des élites portugaises voulant limiter l'émigration légale pour conserver une main-d'œuvre nombreuse et peu payée, même si, au long du xix siècle, ces réticences n'avaient pas empêché une très importante émigration illégale, surtout vers le Brésil. Mais, en 1916, dans le cadre d'une alliance militaire et de l'engagement du CEP, le gouvernement portugais avait accepté de signer, le 28 octobre 1916, un accord de main-d'œuvre avec la France, tout en précisant qu'il serait limité à la durée de la guerre.

L'accord organisait une émigration légale et contrôlée par les deux pays. Le gouvernement portugais avait tenu à limiter les possibilités de recrutement de travailleurs qualifiés, peu nombreux au Portugal, et voulait maintenir à l'écart des recrutements les secteurs industriels nécessaires à son propre effort de guerre. Parallèlement, les pressions des grands propriétaires fonciers exigeraient aussi de limiter les départs de trop nombreux travailleurs agricoles. L'accord prévoyait des recrutements de travailleurs volontaires, avec des contrats de 6 mois renouvelables. Les départs devaient se faire en groupes de 50 à 150 personnes, avec des passeports collectifs, ce qui limitait la liberté des travailleurs. Les autorités françaises prenaient en charge les frais de transport. Et, du fait de la guerre, pour la première fois, les transports se firent par train et non plus par bateau. C'est à partir de la frontière française que les travailleurs portugais étaient affectés dans différentes entreprises, dans diverses régions de France, selon une répartition prévue à l'avance

et communiquée au consul portugais à Bayonne, responsable du " dépôt ». Les premiers groupes de travailleurs portugais arrivèrent à Hendaye dès les derniers mois de 1916. En décembre 1916, 854 travailleurs portugais y étaient déjà passés, affectés dans des usines métallurgiques travaillant pour la guerre, à Givors, Bourges, Sochaux et dans la région de Bayonne. Le bilan statistique total est incertain, mais environ 13000 ouvriers furent recrutés légalement pour des usines et les travaux publics. II faudrait y ajouter ceux qui furent recrutés pour l'agriculture et les travaux forestiers.

Selon l'accord, les conditions de travail et de salaires devaient être à égalité avec celles des Français. Le résultat fut mitigé et beaucoup de Portugais furent déçus par leur travail et leur logement. De plus, ils n'étaient pas habitués aux conditions climatiques qu'ils trouvèrent en France et n'avaient pas de vêtements adaptés. Enfin, ils étaient isolés dans un pays dont ils ne connaissaient pas la langue et où personne ne connaissait la leur. Certains d'entre eux firent

L'accord prévoyait des recrutements de travailleurs volontaires, avec des contrats de 6 mois renouvelables. Les départs devaient se faire en groupes de 50 à 150 personnes, avec des passeports collectifs, ce qui limitait la liberté des travailleurs. connaître leurs plaintes à l'ambassadeur du Portugal. Toutefois, la situation était difficile car les affectations des travailleurs étaient liées à un recrutement dans le cadre de l'effort de guerre et ne pouvaient pas être modifiées sur simple demande. L'accord prévoyait la présence d'un envoyé du gouvernement portugais qui aurait la responsabilité d'être en contact avec les travailleurs et de constater leurs conditions de vie et de travail. Toutefois, cette nomination tarda à s'organiser et le médiateur manqua de moyens pour sa mission. Certains ouvriers parvinrent à changer légalement (et illégalement) d'affectation, dans ces années de très fort besoin de main-d'œuvre en France. Au bout de six mois, une partie des travailleurs portugais ne renouvelèrent pas leur contrat. Mais 


\section{REPÉRAGE}

d'autres les remplacèrent, tant étaient difficiles les conditions de vie et de travail au Portugal, au point que, dans certaines campagnes du sud du pays, des latifundistes usèrent de toutes sortes de pressions pour retenir leurs travailleurs.

Les études sur ces travailleurs en sont encore à leurs débuts et il serait très intéressant de préciser qui furent ceux qui protestèrent, non seulement en fonction des travaux auxquels ils étaient affectés, mais aussi

Au total, entre les entrées

légales et irrégulières,

on évalua à plus de

25000 les travailleurs

portugais qui entrèrent en

France de 1916 à 1918 de leurs traditions de travail et de lutte au Portugal. En effet, dans les deux premières décennies du XXe siècle, les luttes sociales furent nombreuses au Portugal, dans de nombreuses entreprises, comme dans une partie des latifundia de la province de l'Alentejo. II faut remarquer que, parallèlement à ces recrutements légaux de travailleurs, d'autres Portugais qui n'avaient pas pu, ou pas voulu, passer par les règles de l'accord partirent en France, où ils trouvèrent du travail dans cette période de grand besoin de main-d'œuvre. Ainsi, dès 1915, des arrivées de travailleurs portugais en situation illégale sont signalées à Hendaye et Bordeaux. Au total, entre les entrées légales et irrégulières, on évalua à plus de 25000 les travailleurs portugais qui entrèrent en France de 1916 à 1918.

\section{La formation du corps expéditionnaire portugais (CEP)}

Dès 1914, la guerre toucha le Portugal de diverses façons, sur le plan des équilibres économiques ou en fragilisant ses colonies d'Afrique (Angola et Mozambique) frontalières avec des colonies allemandes. Une des principales motivations d'entrée en guerre du Portugal fut la préservation de ses droits sur ses colonies africaines lors des négociations à la fin de la guerre. En même temps, en inscrivant le Portugal dans l'effort de guerre, les dirigeants de la jeune République portugaise (proclamée le 5 octobre 1910) voulaient renforcer leur légitimité au sein des nations européennes.

L'alliance du Portugal avec la Grande-Bretagne était dans la logique de leur alliance séculaire. Quant à l'alliance avec la France, elle tenait à la proximité politique, idéologique et culturelle entre les dirigeants de la jeune République portugaise et ceux de la Troisième République française.

Toutefois, il fallut attendre le 9 mars 1916 pour l'entrée en guerre du Portugal, même si la France était très favorable à cet engagement, du fait des réticences britanniques qui tenaient à la fois aux rivalités coloniales anglo-portugaises en Afrique et à la nécessaire adaptation à la guerre des tranchées d'une armée portugaise jusqu'alors coloniale. L'engagement militaire du Portugal, une exigence du gouvernement portugais, fut fortement appuyé par la France. Mais les 56500 soldats des deux divisions du corps expéditionnaire portugais (CEP) furent placés sous le haut-commandement de l'armée britannique, dans la zone des tranchées du front des Flandres (dans le Pas-de-Calais). Toutefois, un corps d'artillerie lourde portugais ( 1350 hommes du CALP) rejoignit les tranchées tenues par l'armée française.

Les combattants du CEP, après un court entraînement au Portugal, furent acheminés par bateaux depuis Lisbonne jusqu'à Brest (sur des bateaux principalement britanniques et, en appui, français et portugais). Les transports se firent progressivement entre février et octobre 1917. Les soldats portugais rejoignaient ensuite en train la ville d'Aires-sur-la-Lys, en 4 à 6 jours, leur zone de front, entre Béthune et Armentières.

Dans les premiers mois de 1918, les soldats portugais se trouvèrent peu à peu fragilisés par les 
tensions politiques et sociales au Portugal, avec la dictature de Sidónio Pais (décembre 1917-décembre 1918) et les nombreuses oppositions à la guerre. Enfin, les soldats du CEP furent privés de relève et de renforts fin 1917, avec l'entrée en guerre des États-Unis qui mobilisa les navires britanniques au détriment du transport des soldats portugais. En outre, ces derniers ne pouvaient pas prendre leurs permissions au Portugal.

\section{La bataille de la Lys, le 9 avril 1918}

Au terme d'une année de front, sur les 56500 Portugais du CEP, on comptait déjà 1044 morts, une centaine de disparus et près de 3800 blessés. Et, comme dans toutes les armées des pays en guerre, le CEP en 1917 avait connu des découragements, des révoltes, des désertions (372 condamnations, mais une seule à mort pour trahison). La première division portugaise avait été repliée et la seconde division portugaise devait être relayée par des soldats britanniques le 9 avril 1918, le jour où l'armée allemande lança une très grosse offensive pour percer le front des Flandres. La bataille d'Ypres (dont la bataille de la Lys marqua le début) fut un des épisodes terribles de la Première Guerre mondiale. L'offensive allemande n'atteignit pas son but et coûta la vie à 348000 Allemands, 236000 Britanniques et 92 ooo Français. Les Portugais comptèrent 1341 morts, 4626 blessés, 1932 disparus et 7740 prisonniers. Les survivants du CEP furent réorganisés et intégrés à la $5^{\mathrm{e}}$ armée britannique et participèrent ainsi aux derniers combats de 1918. Au total, l'engagement des Portugais dans les tranchées des Flandres leur coûta 2266 morts, 1991 disparus et 12508 blessés.

Dès le début de la guerre, et avant l'entrée en guerre du Portugal, une vingtaine de citoyens portugais s'étaient engagés dans l'armée française et avaient été affectés au $7^{\text {er régiment }}$ de marche de la Légion étrangère. II s'agissait de jeunes gens ayant déjà un bon niveau d'étude, comme en témoignent leurs lettres. En 1916, leur situation était difficile, une partie d'entre eux ayant été blessés. Après l'entrée en guerre du Portugal, ils demandèrent d'être affectés au CEP.

\section{Mémoires partagées françaises et portugaises}

Dès le 14 juillet 1918, une délibération du conseil municipal de Paris décida, " en hommage aux soldats portugais », de baptiser "Avenue des Portugais " l'ex-Avenue de Sofia (capitale d'un pays devenu allié de l'Allemagne). Le 14 juillet 1919, 400 soldats portugais participèrent au défilé de la victoire sur les Champs-Élysées, aux côtés de centaines de soldats alliés. Dans les années 19201930, de nombreuses initiatives mêlèrent Français et Portugais pour des hommages aux combattants portugais.

Des cérémonies annuelles du

À partir de la seconde moitié des années 1970, les cérémonies prirent de l'importance, unissant des associations portugaises, les municipalités françaises concernées et des représentants des autorités portugaises. souvenir se déroulent, dans les jours proches du 9 avril, dans les deux villages qui gardent la mémoire de la bataille de la Lys, c'est-à-dire au cimetière militaire portugais près du village de Richebourg l'Avoué (inauguré en 1935) et au monument en hommage " au soldat portugais " érigé devant l'église du village de La Couture (inauguré en 1923). Quelques tombes de soldats portugais se trouvent aussi dans le grand cimetière militaire britannique à Boulogne. Enfin, sur l'Anneau de la mémoire inauguré à NotreDame-de-Lorette en novembre 2014, figurent 2266 noms de soldats portugais tombés en France. 


\section{REPÉRAGE}

Les cérémonies au monument de La Couture et au cimetière de Richebourg furent interrompues par la Seconde Guerre mondiale. Elles reprirent à la fin des années 1940. Ce furent surtout les associations portugaises de la région qui continuèrent à faire vivre la mémoire des soldats portugais. À partir de la seconde moitié des années 1970, les cérémonies prirent de l'importance, unissant des associations portugaises, les municipalités françaises concernées et des représentants des autorités portugaises.

\section{La Grande Guerre, première étape de l'immigration portugaise en France}

Les bases de l'amorce de l'émigration des travailleurs portugais vers la France ont été mises en place dans le cadre des exigences militaires et économiques de la Grande Guerre. Les premiers travailleurs, en dépit des déceptions, et les exemples des soldats restés en France amorcèrent la première étape de l'immigration portugaise en France.

Durant la guerre, lors de permissions, des soldats portugais avaient participé à certains travaux agricoles dans les zones en arrière du front. D'autres témoignages, lors des cérémonies à La Couture et à Richebourg, rappellent le bon accueil fait aux soldats por-

La décennie 1920 connut une première progression notable de Portugais puisque, en 1931,

ils étaient près de 50000 . tugais par les habitants de Béthune et à ceux restés dans les villages proches du front. Ces relations se renforcèrent après l'armistice du 11 novembre 1918, car la démobilisation des soldats portugais fut très lente et a offert l'occasion de nombreux rapprochements avec la population française, d'autant plus que les soldats durent trouver un emploi localement, dans l'attente de leur rapatriement au Portugal. Après 1919, l'ins- tallation dans la région devient définitive pour certains soldats qui fondèrent des familles en France. En 1928, on comptait plus de 2000 Portugais vivant dans la région de Béthune, Hénin-Liétard, Albert et Vimy, sur près de 50000 Portugais qui furent alors recensés en France.

Alors que l'on y comptait à peine un millier de Portugais en 1914, le recensement de 1921 en dénombrait plus de 10 ooo et, pour la première fois, les statistiques françaises ouvraient une catégorie « Portugais ». La décennie 1920 connut une première progression notable de Portugais puisque, en 1931, ils étaient près de 50000.

Pourtant, cette première étape de l'immigration de travailleurs portugais en France ne s'est pas faite dans la continuité que désiraient les responsables français. En effet, dès 1918 et en prévision de la fin de la guerre, les autorités françaises avaient proposé aux responsables portugais de prolonger la convention de maind'œuvre d'octobre 1916 par un nouvel accord de main-d'œuvre. Et, en 1919, la France proposait au Portugal la signature d'un accord, semblable à ceux signés à cette époque par la France avec la Pologne et l'Italie. Deux missions françaises, I'une du ministère du Travail, l'autre du ministère de l'Agriculture, purent aller au Portugal afin de mieux connaître les possibilités de recrutement de travailleurs portugais. Mais les gouvernements portugais reportèrent toujours la signature d'un tel accord, que ce soit ceux de la République ou ceux de la dictature (après 1926), sous la pression des milieux patronaux industriels et agricoles. Les recrutements se firent donc soit légalement, sous forme de contrats individuels, ou, dans un nombre croissant de cas, illégalement. Or, en l'absence d'un accord de main-d'œuvre, les travailleurs portugais n'étaient pas couverts en cas d'accident, de maladie et de chômage, d'où les nombreuses demandes infructueuses d'immigrants portugais auprès des autorités portugaises en France pour bénéficier de ces protec- 
tions. Toutefois, à la veille de la Deuxième Guerre mondiale, les difficultés au Portugal et la reprise des demandes de main-d'œuvre de la part de la France amenèrent Salazar à accepter de signer un tel accord. Mais ce fut en mai 1940, un mois avant l'invasion et la défaite française. L'accord ne fut donc pas mis en œuvre, même si certaines autorités portugaises cherchèrent à en obtenir une application partielle en matière de protec- tion sociale. II fallut attendre décembre 1963 pour qu'un accord soit signé. Mais les autorités de l'Estado Novo mirent tant de mauvaise volonté à l'appliquer que des centaines de milliers de Portugais émigrèrent illégalement, au point que, à la fin des années 1960, $80 \%$ des arrivées de travailleurs portugais en France furent illégales. II fallut attendre 1971 pour qu'un accord de main-d'œuvre soit signé et respecté, 55 ans après le premier accord !

\section{Quelques références bibliographiques}

Cristina Climaco, «L'exil portugais en France (1927-1931) : corps en exil, esprit au Portugal », in Cahiers de la Méditerranée, $n^{\circ}$ 82, 2011, pp. 79-88.

Jean Derou, Les relations franco-portugaises (1910-1926), Paris, Publications de la Sorbonne, 1986. Laurent Dornel, Les étrangers dans la Grande Guerre, Paris, Musée de l'histoire de l'immigration/ La documentation française, 2014.

Yvette Dos Santos, «L'État portugais face à l'émigration et aux impératifs de la Première guerre mondiale ", in Exils et migrations ibériques au XXe siècle, $n^{\circ}$ 5, 2013.

Léonard Yves, Le Portugal au XXe siècle, Paris, Chandeigne, 2016.

Marie-Claude Munoz, "Le Corps expéditionnaire portugais, 1916-1918 », in Hommes \& Migrations, $n^{\circ} 1148,1991$, pp. 15-18.

Marie-Claude Munoz, « Les relations franco-portugaises, 1916-1918 », in Revista Mosaico, vol. 2, $\mathrm{n}^{\circ}$ 1, 2009, pp. 68-72.

Victor Pereira, «Émigration et politique de main-d'œuvre au Portugal (1957-1974) », in Hommes \& Migrations, n 1263, pp. 58-69.

Marie-Christine Volovitch-Tavares, « La Grande Guerre et l'amorce de l'immigration des Portugais en France ", in Marie-Claude Blanc-Chaléard, Anne Dulphy, Marie-Anne Matard-Bonucci (dir.), D'Italie et d'ailleurs. Mélanges en hommage à Pierre Milza, Rennes, Presses universitaires de Rennes, 2014, pp. 105-116.

Marie-Christine Volovitch-Tavares, 100 ans d'histoire des Portugais en France, Paris, Michel Lafon, 2016.

Trois colloques réalisés en 2016, à Hendaye (28 octobre), à Bordeaux (25 novembre) et à Paris (10 décembre), ont été consacrés au centenaire de l'entrée en guerre du Portugal et du premier accord de main-d'œuvre entre la France et le Portugal. Les actes seront publiés en 1917 par les éditions Quatorze (coordination de Manuel Dias Vaz), avec les communications de Cristina Climaco, Laurent Dornel, Yvette Dos Santos, Victor Pereira et Marie-Christine Volovitch-Tavares. 